\title{
Exact least-volume trusses for two symmetric point loads and unequal permissible stresses in tension and compression
}

\author{
Tomasz Sokół • George I. N. Rozvany
}

Received: 18 October 2012 / Revised: 19 October 2012 / Accepted: 19 October 2012 / Published online: 8 December 2012

(C) The Author(s) 2012. This article is published with open access at Springerlink.com

\begin{abstract}
In this Authors' Reply previously presented solutions are extended to unequal permissible stresses in tension and compression, comments are made on the lack of adjoint displacement fields in so-called O-regions (memberless regions), and some computational details are explained.
\end{abstract}

Keywords Exact topology optimization · Michell trusses . Optimal regions $\cdot$ Stress constraints

\section{Introduction}

The aim of this contribution is to comment on a Discussion by Vázquez Espi (2013a), subsequently called 'Writer', of a paper by Sokół and Rozvany (2012), subsequently called 'Authors'.

\section{Validity of the Authors' solutions for unequal permissible stresses in tension and compression}

The Writer raises a very interesting point in his Discussion, stating that the Authors' optimal layouts can be extended

\footnotetext{
T. Sokół (凶)

Department of Structural Mechanics and Computer Aided

Engineering, Faculty of Civil Engineering,

Warsaw University of Technology,

Al. Armii Ludowej 16, 00-637 Warsaw, Poland

e-mail: t.sokol@il.pw.edu.pl

G. I. N. Rozvany

Department of Structural Mechanics,

Budapest University of Technology and Economics,

Müegyetem rkpt. 3, Kmf. 35, 1521 Budapest, Hungary

e-mail: smo.rozvany@t-online.hu
}

to unequal permissible stresses in tension and compression. Indeed, this is the case if we have 'statically determinate' support conditions, more specifically a hinge (pin) and a roller support. For such support conditions a layout based on Michell's (1904) original optimality criteria is also valid for unequal permissible stresses (see e.g. the paper by Rozvany 1996). Using the current terminology and notation, Michell criteria can be stated as

$$
\begin{array}{ll}
\bar{\varepsilon}=\left(1 / \sigma_{P}\right) \operatorname{sgn} F & (\text { for } F \neq 0), \\
|\bar{\varepsilon}| \leq 1 / \sigma_{P} & (\text { for } F=0),
\end{array}
$$

where $\sigma_{P}$ denotes the permissible stress in both tension and compression, $\bar{\varepsilon}$ represents a fictitious strain-field termed 'adjoint' strain field, and $F$ is the longitudinal force in any truss member.

However, if the support conditions are statically indeterminate (as in the case of two pin supports), then for unequal permissible stresses we must use the modified optimality conditions (see e.g. the papers by Rozvany 1996, or Sokó and Rozvany 2012 or Rozvany and Sokół 2012)

$$
\begin{array}{ll}
\bar{\varepsilon}=-1 / \sigma_{C} & (\text { for } F<0), \\
\bar{\varepsilon}=1 / \sigma_{T} & (\text { for } F>0), \\
-1 / \sigma_{C} \leq \bar{\varepsilon} \leq 1 / \sigma_{T} & (\text { for } F=0),
\end{array}
$$

where $\sigma_{T}$ and $\sigma_{C}$ denote the permissible stresses in tension and compression.

Note: The Writer is absolutely correct in stating in his Discussions (see also Vázquez Espi 2013b) the following.

(i) Trusses with statically indeterminate support conditions, requiring the modified optimality conditions in (2), should not be termed 'Michell structures' (the Authors call them 'generalized Michell structures' in recent publications). 
(ii) For 'Michell structures' (i.e. trusses with given external forces, or equivalently with statically determinate supports) the Authors' optimal layouts are also valid for unequal permissible stresses in tension and compression. Moreover, it follows from symmetry principles (Rozvany 2011a), that these solutions are symmetrical about the horizontal axis.

(iii) For statically indeterminate supports, the reaction forces and their cost depend on the choice of truss topology, and therefore the reaction (support) cost should be taken into consideration in any realistic truss optimization. This is attempted in the Authors' recent paper (Rozvany and Sokół 2012).

It will be shown in two examples that for statically indeterminate support conditions the modified optimality conditions in (2) give a much lower volume than Michell's classical criteria. Naturally, for these support conditions (in our case, two pin supports), the optimal layout is in general different from the ones in the Authors' paper (Sokół and Rozvany 2012), and it depends on the $\sigma_{T} / \sigma_{C}$ ratio.

\subsection{Example 1: one point load between two supports, unequal permissible stresses in tension and compression}

The considered example can be regarded as a special (limiting) case of a truss with two point loads, in which the distance between the loads tends to zero. This problem was fully discussed in a recent paper (Rozvany and Sokół 2012). The optimal topology for a pin and a roller support is shown in Fig. 1a, and the one for two pin supports in Fig. 1b. The optimal layout in Fig. 1a is actually due to Michell (1904), but he considered given external forces instead of supports. As the Writer states, the solution in Fig. 1a (with statically determinate supports) is valid for any stress ratio $\sigma_{T} / \sigma_{C}$. For the layout in Fig. 1b (with statically indeterminate supports) the angle $\alpha$ depends on the stress ratio. For $\sigma_{T} /$ $\sigma_{C}=3$, we get $\alpha=30^{\circ}$ (see Rozvany and Sokół 2012).

The optimal truss volume for the pin-roller support is (Fig. 1a)

$V_{\text {pin-roller }}=P L(4+2 \pi) / \sigma_{T}=10.283185 P L / \sigma_{T}$.

The optimal volume for the pin-pin support is (Fig. 1b)

$V_{\text {pin-pin }}=P L(4 \pi / 3+2 \sqrt{3}) / \sigma_{T}=7.652892 P L / \sigma_{T}$,

hence the pin-roller support has $34.4 \%$ bigger optimal truss volume than the pin-pin support.

The same substantial difference in volume exists between solutions calculated by Michell's original optimality criteria in (1) and by the modified conditions in (2).
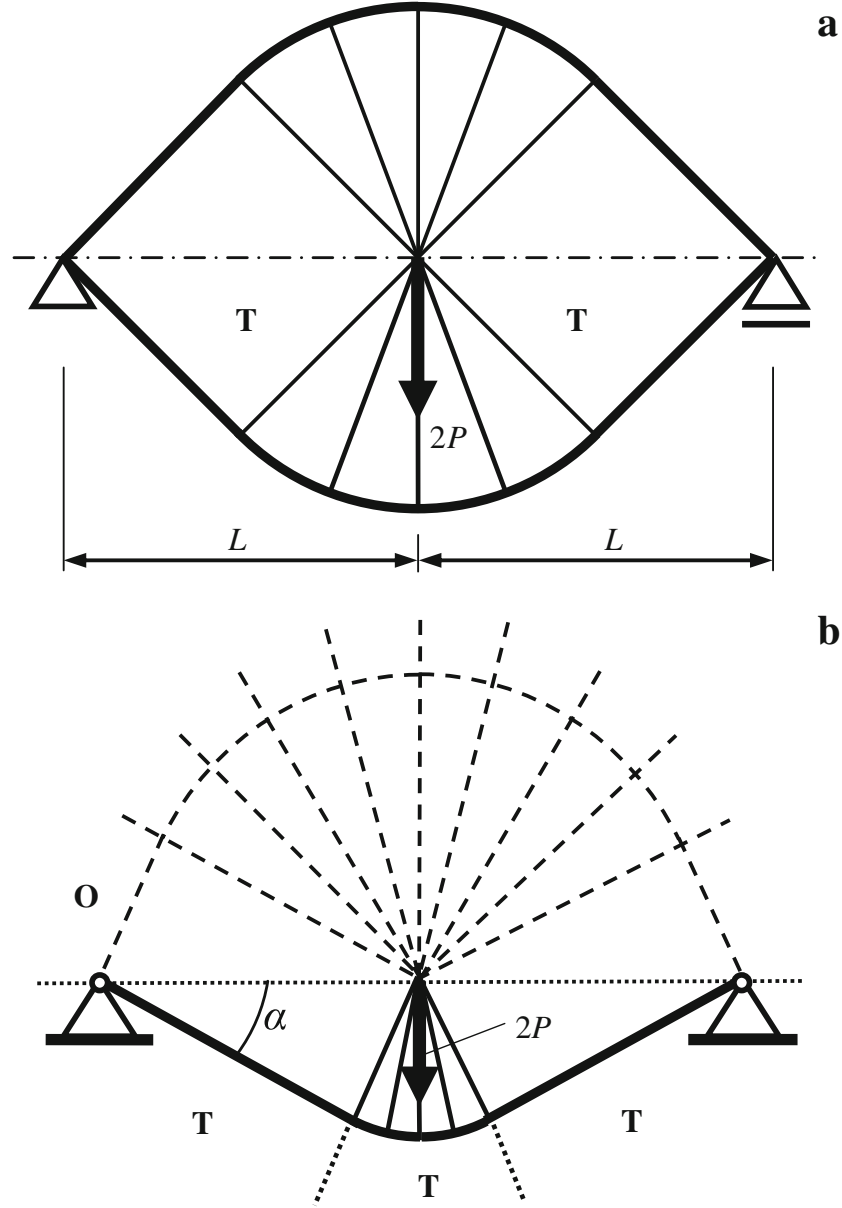

b

Line notation:

c

$$
\begin{aligned}
& \text { principal adjoint strains } \\
& \text { in the O-region } \\
& \text { region boundaries } \\
& \text { 'concentrated' members } \\
& \text { 'distributed' members }
\end{aligned}
$$

Fig. 1 Optimal topologies for a single point load and unequal permissible stresses in tension and compression: a pin and roller supports, b two pin supports, $\mathbf{c}$ line notation

\subsection{Numerical confirmation of the optimal solutions} in Example 1

The numerical solutions corresponding to the analytical solutions in Fig. 1 are shown in Fig. 2. They have the volumes

$$
\begin{aligned}
V_{\text {pin-roller }} & =10.286269 P L / \sigma_{T}, \\
V_{\text {pin-pin }} & =7.6538106 P L / \sigma_{T} .
\end{aligned}
$$



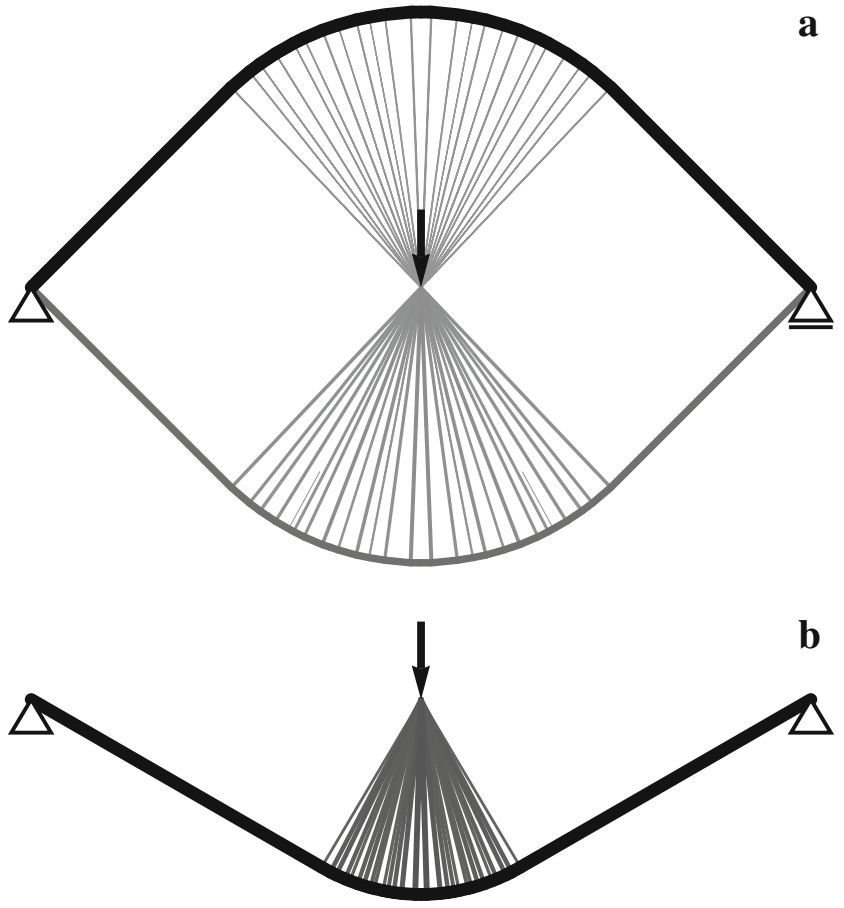

Fig. 2 Numerical solutions for the optimal topologies in Fig. 1

The number of potential truss elements used for the numerical solutions were 221 million (Fig. 2a) and 1.97 billion (Fig. 2b), resulting in relative errors of $0.03 \%$ and $0.01 \%$ compared to the analytically calculated optimal volumes in (3) and (4). These numerical solutions are more accurate than those published previously (Rozvany and Sokół 2012).

\subsection{Example 2: two point loads between supports, unequal permissible stresses in tension and compression}

The loading and support conditions, together with the exact optimal truss topology for equal permissible stresses in tension and compression and either two pin supports or a pin and a roller support (Sokół and Rozvany 2012) can be seen in Fig. 3a, which shows one quarter of the domain. For unequal permissible stresses and a pin and a roller support the optimal topology is the same. Then the optimum truss volume for a stress ratio of $\sigma_{T} / \sigma_{C}=3$ becomes

$$
\begin{aligned}
V_{\text {pin-roller }} & =P h[\sqrt{2}(4+2 \pi)+4] / \sigma_{T} \\
& =18.542620126 P h / \sigma_{T} .
\end{aligned}
$$

A numerical solution with more than one billion potential members (Fig. 3b) fully confirmed the optimal layout in Fig. 3a and gave the volume value of

$V_{\text {pin-roller }}=18.545645 \mathrm{Ph} / \sigma_{T}$

with a relative error of only $0.016 \%$.
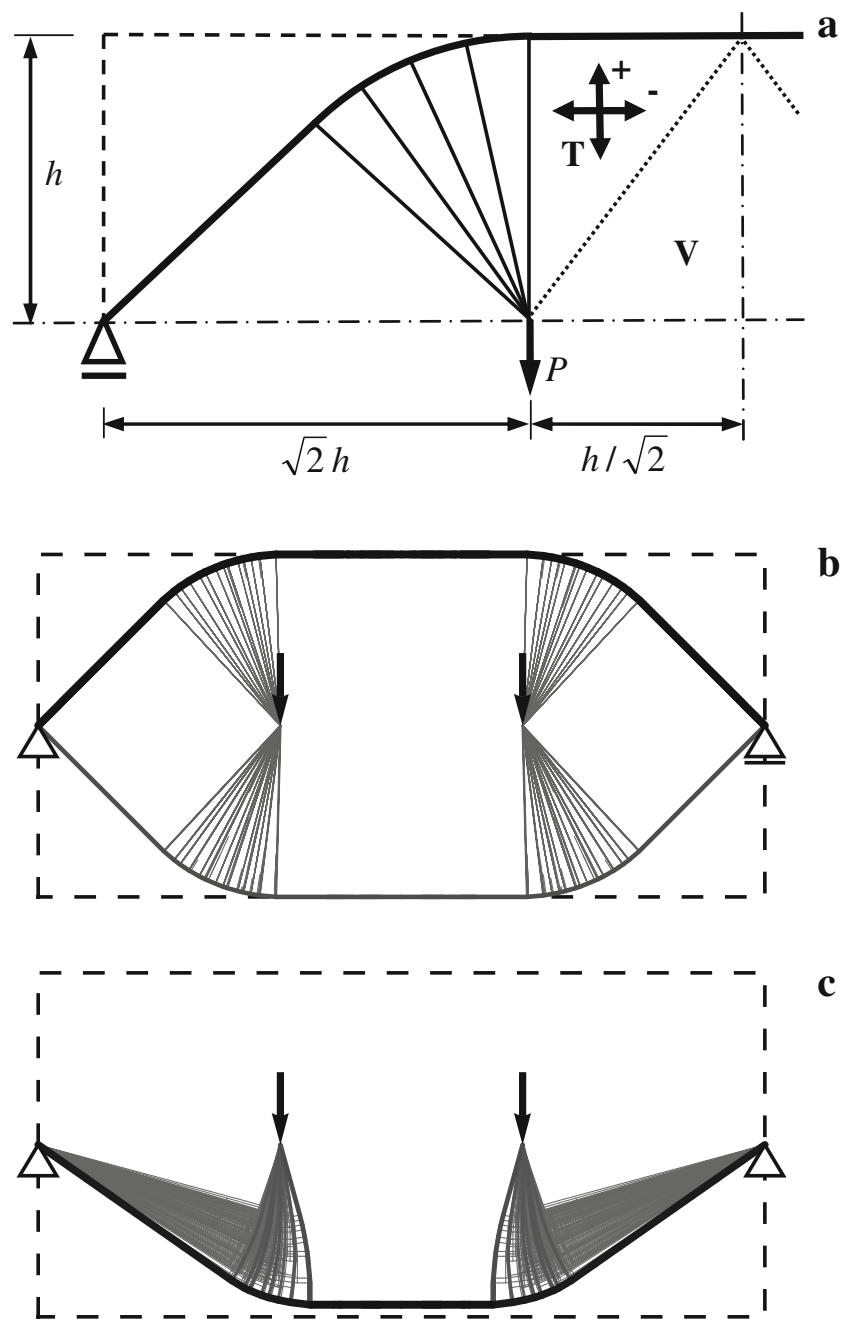

Fig. 3 Optimal topologies for two point loads: analytical solution a and numerical solution $\mathbf{b}$ for a pin and a roller support, numerical solution for two pin supports $\mathbf{c}$

For a stress ratio of $\sigma_{T} / \sigma_{C}=3$ and pin-pin supports the analytical solution is not available as yet (the Authors are working on it), but a numerical solution (Fig. 3c) for the same ground structure as before has given the volume of

$V_{\text {pin-pin }}=13.546874 P h / \sigma_{T}$,

which is $26.9 \%$ smaller than the volume for the pin-roller support in (4).

Summarizing, examples with both one and two point loads have confirmed that for supports with a pin and a roller the optimal topology does not depend on the $\sigma_{T} / \sigma_{C}$ ratio. However, for two pin supports, there is a significant dependence of both optimal topology and optimal volume on the stress ratio value.

It is to be remarked that Pichugin et al. (2012) have also found that for a pin-pin support the optimal topology 
depends on the $\sigma_{T} / \sigma_{C}$ ratio, in their case for a uniformly distributed load between supports.

\section{Lack of optimal adjoint strain fields for some O-regions in the Authors' paper}

The Writer is completely correct in saying that the analytical proof of some solutions in the Authors' paper is incomplete, because the adjoint strain field for some O-type (memberless) regions has not been determined.

The Authors would only like to point out that they are in the process of finding additional optimal solutions with optimal adjoint strain fields in O-regions. Examples can be seen in Figs 2 and 3 of the Authors' paper (Sokół and Rozvany 2012).

Moreover, numerical calculations with over a billion potential elements have shown an excellent (four-digit) agreement with the analytically derived optimal truss volume. Also, it has been found in the past that derivation of the adjoint displacement field for all regions with members usually ensures optimality of the topology.

Summarizing, the Authors completely agree with the Writer on this issue too.

\section{Explanation of the numerical code used in the considered paper}

The program written by the first Author makes use of the 'adaptive ground structure' approach, proposed by Gilbert and Tyas (2003) but applies different strategy of addingremoving of the active bars (Sokół 2011a, b). This approach can also be classified as the special case of the active set method for solving large-scale truss optimization problem for so-called 'plastic layout design' (the latter was introduced by Prager and Rozvany 1977). Furthermore, the solutions of optimization subproblems occurring in successive iterations are achieved by using the fast interior point method with sparse matrix representation (see Sokół 2011a). It should be noted that despite the iterative character of the solution method, it is still formulated as a linear programming optimization problem and therefore the convergence to global optimum is assured. The result of the proposed program is a least-volume truss transmitting given loading to given supports for the prescribed design domain discretized by an appropriate ground structure.

For full plane or half plane problems, the discretization is usually performed several times. At the beginning it is recommended to apply a relatively coarse ground structure with a wide rectangular domain, just to get an idea about the optimal layout and to determine a better adjusted domain for future (denser) discretization. To do this effectively, and to make the design domain as small as possible, we can apply the augmentation and reduction theorem, recently developed by Rozvany (2011b). It is rather rare to have such comfortable situations as in Figs. 5 and 6 of Sokół and Rozvany (2012), for which we knew the exact solutions. In such cases we can adjust the rectangular domain very precisely and this is the reason for the high 'densities' of ground structures applied in the second column in Table 1 of the above paper. The word 'density' was used unfortunately there because the size of the base cell was the same for all cases in Fig. 5 of Sokół and Rozvany (2012). From this point of view we should say that the density was constant, only the size of ground structure was adjusted (for computational efficiency).

In general it is true that denser discretization gives a more accurate solution but this is not the case for results in Figs. 5 or 6 of Sokół and Rozvany (2012) due to different rectangular domains. Of course, if the design domain is fixed and we apply different ground structures for it, the denser mesh and therefore greater number of potential bars usually gives a better result. However, the dependence of the quality of the solution on the number of bars may be more complicated.

A different explanation is required for the ground structures used in Fig. 6 (Sokól and Rozvany 2012), with the horizontal division of distance $L$ into 169 cells. This (somewhat strange) number was adopted for obtaining a good approximation of the position of the applied force defined by the quotient $d / L=\sqrt{2} /(1+\sqrt{2}) \approx 0.585786$, which was approximated by $99 / 169 \approx 0.585799$. (Note that the latter number is greater. This was done intentionally for the problem of Fig. 6 (Sokół and Rozvany 2012) to assure that the numerical volume is not smaller than the exact one due to improper positioning of the load-in this case the force closer to support gives a smaller volume). In their original paper, the Authors assumed that such a detailed explanation is not necessary, because the aim of the paper was to present more general principles.

\section{Concluding remarks}

The Authors wish to thank the Writer for an interesting and useful Discussion, which has given them an opportunity to clarify certain issues related to their paper.

Acknowledgement Financial support from OTKA (Grant No. K 81185 ) is gratefully acknowledged.

Open Access This article is distributed under the terms of the Creative Commons Attribution License which permits any use, distribution, and reproduction in any medium, provided the original author(s) and the source are credited. 


\section{References}

Gilbert M, Tyas A (2003) Layout optimization of large-scale pinjointed frames. Eng Comput 20:1044-1064

Michell AGM (1904) The limits of economy of material in frame structures. Philos Mag 8:589-597

Pichugin AV, Tyas A, Gilbert M (2012) On the optimality of Hemp's arch with vertical hangers. Struct Multidisc Optim 46:17:25

Prager W, Rozvany GIN (1977) Optimization of the structural geometry. In: Bednarek AR, Cesari L (eds) Dynamical systems (Proc. int. conf. Gainsville, Florida). Academic Press, New York, pp 265-293

Rozvany GIN (1996) Some shortcomings of Michell's truss theory. Struct Optim 12:244-250

Rozvany GIN (2011a) On symmetry and non-uniqueness in exact topology optimization. Struct Multidisc Optim 43:297-317

Rozvany GIN (2011b) Domain augmentation and reduction in structural topology optimization. Struct Multidisc Optim 44: 589-591

Rozvany GIN, Sokół T (2012) Exact truss topology optimization: allowance for support costs and different permissible stresses in tension and compression-extensions of a classical solution by Michell. Struct Multidisc Optim 45:367-376

Sokół T (2011a) A 99 line code for discretized Michell truss optimization written in Mathematica. Struct Multidisc Optim 43:181190

Sokół T (2011b) Topology optimization of large-scale trusses using ground structure approach with selective subsets of active bars. In: Borkowski A, Lewiński T, Dzierżanowski G (eds) 19th int conf on 'computer methods in mechanics', CMM 2011, 09-12 May, Warsaw, Book of Abstract, pp 457-458

Sokół T, Rozvany GIN (2012) New analytical benchmarks for topology optimization and their implications. Part I: bi-symmetric trusses with two point loads between supports. Struct Multidisc Optim 46:477-486. doi:10.1007/s00158-012-0786-4

Vázquez Espi M (2013a) New analytical benchmarks for topology optimization and their implications: comments and questions. Struct Multidisc Optim. doi:10.1007/s00158-12-825-1 (to be published in the same issue as this Authors' Reply)

Vázquez Espi M (2013b) On allowance for support costs in the Prager-Rozvany layout theory. Struct Multidisc Optim (accepted). doi:10.1007/s00158-012-850-0 